\title{
Necessity of Dark Energy from Thermodynamic Arguments
}

\author{
H. Moradpour, ${ }^{1}$ A. Sheykhi, ${ }^{1,2}$ N. Riazi, ${ }^{3}$ and B. Wang ${ }^{4}$ \\ ${ }^{1}$ Physics Department and Biruni Observatory, College of Sciences, Shiraz University, Shiraz 71454, Iran \\ ${ }^{2}$ Research Institute for Astronomy and Astrophysics of Maragha (RIAAM), P.O. Box 55134-441, Maragha, Iran \\ ${ }^{3}$ Physics Department, Shahid Beheshti University, Evin, Tehran 19839, Iran \\ ${ }^{4}$ INPAC and Department of Physics, Shanghai Jiao Tong University, Shanghai 200240, China
}

Correspondence should be addressed to A. Sheykhi; asheykhi@shirazu.ac.ir

Received 17 August 2013; Accepted 26 December 2013; Published 20 January 2014

Academic Editor: Kishor Adhav

Copyright (C) 2014 H. Moradpour et al. This is an open access article distributed under the Creative Commons Attribution License, which permits unrestricted use, distribution, and reproduction in any medium, provided the original work is properly cited. The publication of this article was funded by SCOAP ${ }^{3}$.

Considering the cosmic fluid as a quasi-static thermodynamic system, the status of the generalized second law of thermodynamics is investigated and the valid range of the equation of state parameter is derived for a few important cosmological models. Our study shows that the satisfaction of the laws of thermodynamics in these cosmological models requires the existence of some kind of energy in our universe with $\omega<-1 / 3$. In other words, the existence of a dark energy component, or equivalently modified gravity theory, is unavoidable if the cosmological model is to approach thermal equilibrium in late times.

\section{Introduction}

Thermodynamical nature of Einstein's theory of general relativity was first disclosed by Jacobson [1] who showed that the hyperbolic second order partial differential field equations of gravity can be derived by applying the first law of thermodynamics on any local Rindler horizon. Generalization of this method to $f(R)$ gravity, by introducing the entropy generation term due to nonequilibrium nature of spacetime, was investigated in [2]. More attempts to reveal the connection between thermodynamics and various theories of gravity can be found in [3-8]. An elegant example is the derivation of the Friedmann equations as a consequence of the validity of the first law of thermodynamics on the apparent horizon of the Friedmann-Robertson-Walker (FRW) universe [9].

Recently, an entropic origin for gravity was proposed by Verlinde [10]. He argued that the laws of gravity are not fundamental and in particular they emerge as an entropic force caused by the changes in the information associated with the positions of material bodies. Verlinde's derivation of Newton's law of gravitation at the very least offers a strong analogy with the well-understood statistical approach. Therefore, this derivation opens a new window to understanding gravity from first principles. The entropic approach to gravity has arisen a lot of enthusiasm, recently (see, e.g.,
[11-34] and references therein). The studies have also been generalized to higher order gravity theories such as GaussBonnet and Lovelock gravity [35]. All these attempts indicate a hope to achieve a deeper connection between gravity and thermodynamics.

From the second law of thermodynamics, we know that every closed system moves towards its maximum entropy state which is an equilibrium state. This leads to the conclusion that the second derivative of the entropy should be negative [36]. The assumption that the second derivative of entropy is negative comes from the fact that while the entropy is increasing as the system approaches equilibrium, it should tend to a maximum; hence the first derivative which is already positive tends to zero, leading to a negative second derivative. Indeed, our discussion in this paper is based on that the natural tendency of systems to evolve toward thermodynamical equilibrium is characterized by two properties of its entropy function, $S(x)$; namely, it is always a nondecreasing function, $d S(x) / d x \geq 0$, and is a convex function, $d^{2} S(x) / d x^{2} \leq 0$ [37]. In the context of cosmology, this implies that the total entropy including the entropy associated with the apparent horizon together with the matter field entropy inside the apparent horizon must be a nondecreasing function of scale factor, $S^{\prime}(a) \geq 0$, where the 
prime stands for the derivative with respect to scale factor $a$. This statement is usually called the generalized second law (GSL) of thermodynamics. In addition as $a \rightarrow \infty$ we must have $S^{\prime \prime}(a) \leq 0$. Applying these requirements to the cosmological setup leads to interesting constraints on the equation of state parameter of the cosmic fluid filling the universe.

We consider a background which is filled by a homogeneous fluid with the equation of state $p=\omega \rho$ and endorsing situations in that the background component of the total entropy plays the role of dominate entropy in fulfilling the GSL of thermodynamics in the long run limit. In $[38,39]$, authors have divided the entropy of the universe into three parts arising from the matter field, geometry (horizon), and an unknown fluid with the equation of state $p=\omega \rho$.

Then, they studied situations in which the background component of entropy has a major contribution to the total entropy of the universe and that it satisfies GSL. In this way, the authors of $[38,39]$ could find the proper range of $\omega$ which is consistent with dark energy. In the present work, we do not separate matter component from the dominant fluid and try to reach the corresponding implications for the equation of state in various theories of gravity. Further calculations using this approach were presented in [38, 39].

Let us have a glimpse at general properties of a homogeneous and isotropic FRW universe which is described by the line element

$$
d s^{2}=h_{\mu \nu} d x^{\mu} d x^{\nu}+\widetilde{r}^{2}\left[d \theta^{2}+\sin ^{2} \theta d \phi^{2}\right]
$$

where $\tilde{r}=a(t) r$ and $h_{\mu \nu}=\operatorname{diag}\left(-1, a^{2}(t) /\left(1-k r^{2}\right)\right)$ with $x^{0}=t$ and $x^{1}=r$ and $k$ is spatial curvature taking the values $0,-1$, and 1 for a flat, open, and closed universe, respectively [40]. Here $a(t)$ is the scale factor which carries the effect of expansion on the spatial degrees of freedom. Recent observations have confirmed an accelerating universe (i.e., $\dot{a} \geq 0$ and $\ddot{a} \geq 0$ ) [41-43]. The validity of the first condition $(\dot{a} \geq 0)$ is clear. Using the fact that $H=\dot{a} / a$, we have

$$
\ddot{a}=a H\left(H+a H^{\prime}\right),
$$

where the prime stands for derivative with respect to the scale factor $a$. For every model of cosmology this relation makes an upper bound on the equation of state parameter $\omega$ and so must be evaluated for each model separately. The apparent horizon which is defined as a marginally trapped surface with vanishing expansion can be determined by relation $h_{\mu \nu} \partial^{\mu} \widetilde{r} \partial^{\nu} \widetilde{r}=0$. A simple calculation yields

$$
\tilde{r}_{A}=\frac{1}{\sqrt{H^{2}+k / a^{2}}} .
$$

Suppose that the energy-momentum tensor of the total matter and energy in the universe has the form of a perfect fluid, $T_{\mu \nu}=p g_{\mu \nu}+(\rho+p) U_{\mu} U_{\nu}$, where $U^{\nu}$ denotes the fourvelocity of the fluid and $\rho$ and $p$ are the total energy density and pressure of the fluid, respectively. The energy conservation law $\nabla_{\mu} T^{\mu \nu}=0$ leads to the continuity equation in the form

$$
\dot{\rho}+3 H(\rho+p)=0 .
$$

Also, we suppose the equation of state as

$$
p=\omega \rho
$$

where, for radiation and byronic matter, we have $\omega \geq 0$. From the continuity (4), one finds

$$
\rho=\rho_{0} a^{-3(1+\omega)} .
$$

In the long run where $a \rightarrow \infty$, the energy density goes to zero provided that $\omega>-1$. For $\omega<-1$, that is, ghost matter, we have the unusual situation where density grows with expansion. Throughout this paper, we assume a spatially flat FRW spacetime. In the next section, we study thermodynamic nature of ghost dark energy model. Cyclic model, Horava-Liftshitz deformed model, DGP model, and GaussBonnet model will be discussed in the subsequent sections. The last section is devoted to our concluding remarks.

\section{Ghost Dark Energy}

A new dark energy model called "ghost dark energy" was recently proposed to explain the observed accelerating expansion of the Universe [44-51]. In this model, dark energy originates from the Veneziano ghost of QCD [52, 53] with an energy density which is proportional to the Hubble parameter, $\rho_{D}=\alpha H$, where $\alpha$ is a constant of order $\Lambda_{\mathrm{QCD}}^{3}$ or QCD mass scale. Taking into account the fact that $\Lambda_{\mathrm{QCD}} \sim$ $100 \mathrm{MeV}$ and $H \sim 10^{-33} \mathrm{eV}$ for the present time, this gives the right order of magnitude $\rho_{D} \sim\left(3 \times 10^{-3} \mathrm{eV}\right)^{4}$ for the ghost energy density [44]. In this section we would like to constraint the equation of state parameter of ghost dark energy using the entropy argument. Let us rewrite the ghost energy density as [44]

$$
H=\alpha^{-1} \rho .
$$

Substituting (6) and (7) in (2), after simple calculations, we find $\omega \leq-1 / 3$ in order to have an accelerating universe; namely, $\ddot{a} \geq 0$. Assuming the background is filled by a typical fluid with energy density (7), we want to see under which circumstances the GSL is preserved for the universe filled with ghost dark energy. The entropy associated with the apparent horizon in a flat FRW universe obeys the wellknown area law [54]:

$$
S_{h}=\frac{A}{4}=\pi H^{-2}=\pi \alpha^{2} \rho_{0}^{-2} a^{6(1+\omega)},
$$

where $A=4 \pi H^{-2}$ is the apparent horizon area. Throughout this paper, we employ units in which $G=c=\hbar=k_{B}=$ 1. Taking the first and the second derivative of the entropy function $S_{h}$ with respect to the scale factor, we get

$$
\begin{gathered}
S_{h}^{\prime}=6 \pi \alpha^{2} \rho_{0}^{-2}(\omega+1) a^{6 \omega+5}, \\
S_{h}^{\prime \prime}=36 \pi \alpha^{2} \rho_{0}^{-2}(\omega+1)\left(\omega+\frac{5}{6}\right) a^{6 \omega+4} .
\end{gathered}
$$


Hence, we arrive at

$$
\begin{gathered}
S_{h}^{\prime} \geq 0 \Longrightarrow \omega \geq-1, \\
S_{h}^{\prime \prime} \leq 0 \Longrightarrow-1 \leq \omega \leq-\frac{5}{6} .
\end{gathered}
$$

Thus, for both conditions to be satisfied simultaneously, we must have $-1 \leq \omega \leq-5 / 6$ which is in agreement with the accelerating condition $\omega \leq-1 / 3$. Next, we consider the entropy of the perfect fluid inside the horizon. The entropy of the universe inside the horizon $S_{f}$ can be related to its energy and pressure in the horizon by the Gibbs equation [55]:

$$
T_{f} d S_{f}=d E_{f}+p_{f} d V
$$

where $E_{f}$ is the energy of the fluid within the Hubble horizon with radius $L=H^{-1}$,

$$
E_{f}=\rho_{f} V=\frac{4 \pi H^{-3} \rho_{f}}{3}=\frac{4 \pi \alpha^{3}}{3 \rho_{f}^{2}}
$$

where we have also used (7). Therefore, we have

$$
T_{f} \frac{d S_{f}}{d a}=\frac{d}{d a}\left(\frac{4 \pi \alpha^{3}}{3 \rho_{f}^{2}}\right)+\omega \rho_{f} \frac{d V}{d a}
$$

The evolution of the temperature of the matter field can be determined by $\left(d \ln T_{f} / d \ln a\right)=-3 \omega$, which leads to $T_{f}=$ $T_{0} a^{-3 \omega}$ [56]. From (13) one finds

$$
S_{f}^{\prime}=\frac{4 \pi \rho_{0} \alpha^{3}}{3 T_{0}}(\omega+1)\left(6 a^{9 \omega+5}+\frac{9(\omega-1)}{2} a^{12 \omega+8}\right),
$$

where we have used the fact that $\rho=\rho_{f}$. The second derivative of the fluid entropy can be obtained as

$$
\begin{aligned}
S_{f}^{\prime \prime}= & \frac{4 \rho_{0} \pi \alpha^{3}}{3 T_{0}}(\omega+1) \\
& \times\left[6(9 \omega+5) a^{9 \omega+4}+\frac{9(\omega-1)(12 \omega+8)}{2} a^{12 \omega+7}\right] .
\end{aligned}
$$

The ratio between derivatives of different components, the entropy of the fluid, and the entropy associated with the Hubble horizon can be obtained in the long run limit as

$$
\frac{S_{f}^{\prime}}{S_{h}^{\prime}} \approx \frac{S_{f}^{\prime \prime}}{S_{h}^{\prime \prime}} \sim a^{3 \omega}+a^{6 \omega+3} \Longrightarrow \omega<-\frac{1}{2},
$$

which implies that $-3 / 2<\omega<-1 / 2$. By comparing with (10), it becomes obvious that the domain $-1 \leq \omega \leq-5 / 6$ is an appropriate domain for the equation of state parameter of ghost dark energy which satisfies all thermodynamic conditions simultaneously.

\section{Cyclic Universe Model}

The modified Friedman equation of cyclic universe, which can be obtained from the effective theory of loop quantum cosmology, can be written as $[57,58]$

$$
H^{2}=\frac{8 \pi G}{3} \rho\left(1-\frac{\rho}{\rho_{c}}\right)
$$

where $\rho_{c} \approx 10^{76}(\mathrm{GeV})^{4}$ is the critical density, set by quantum gravity [59] and disparate from the usual critical density $3 M_{p}^{2} H^{2}$. Using (2), (6), and (17) in the late time (low density), the upper bound for $\omega$ can be obtained as $\omega \leq-1 / 3$, similar to what we found for ghost dark energy model. Using area law for the horizon entropy, $S_{h}=A / 4=\pi H^{-2}$, as well as Friedmann (17), the first derivative of the entropy with respect to scalar factor reads

$$
S_{h}^{\prime}=\frac{9(\omega+1)}{8 G} \frac{\rho-2 \rho^{2} / \rho_{c}}{a\left(\rho-\rho^{2} / \rho_{c}\right)^{2}} .
$$

In two cases $S_{h}^{\prime}$ can be positive. First for $\rho>\rho_{c} / 2$ and $\omega<-1$, and second for $\rho<\rho_{c} / 2$ and $\omega>-1$. For the late time cosmology where $a \rightarrow \infty(\rho \rightarrow 0)$, (18) reduces to

$$
S_{h}^{\prime} \approx(\omega+1) a^{3(\omega+(2 / 3))} .
$$

Therefore, in order to have $S_{h}^{\prime} \geq 0$ the equation of state parameter should satisfy $\omega \geq-1$. Now, we consider the second derivative of the entropy:

$$
\begin{aligned}
S_{h}^{\prime \prime}= & \frac{9(\omega+1)}{8 G a^{2}\left(\rho-\rho^{2} / \rho_{c}\right)^{2}} \\
\times & {\left[-3\left(\omega+\frac{4}{3}\right) \rho+12(\omega+1) \frac{\rho^{2}}{\rho_{c}}+\frac{2 \rho^{2}}{\rho_{c}}\right.} \\
& \left.+6(\omega+1) \frac{\left(\rho-2 \rho^{2} / \rho_{c}\right)^{2}}{\rho-\rho^{2} / \rho_{c}}\right] .
\end{aligned}
$$

In the late time where $\rho \rightarrow 0$, the above relation can be simplified as

$$
S_{h}^{\prime \prime} \approx(\omega+1)(3 \omega+2) a^{3 \omega+1}
$$

which indicates that $-1 \leq \omega \leq-2 / 3$ for which we have $S_{h}^{\prime \prime} \leq$ 0 . Next, we turn to the calculation of the entropy of a fluid which fills the background spacetime. Following the method of the previous section, we arrive at

$$
\begin{aligned}
S_{f}^{\prime}= & \frac{\rho_{0}(\omega+1) 2 \pi}{(8 \pi G / 3)^{3 / 2} T_{0} a^{4}\left(\rho-\rho^{2} / \rho_{c}\right)^{3 / 2}} \\
& \times\left[3 \omega \frac{\rho-2 \rho^{2} / \rho_{c}}{\rho-\rho^{2} / \rho_{c}}+\frac{3}{\rho_{c}\left(1-\rho / \rho_{c}\right)}+1\right] .
\end{aligned}
$$


One can easily check that at the late time the above relation reduces to

$$
S_{f}^{\prime} \longrightarrow(\omega+1)(3 \omega+1) a^{9 \omega / 2+1 / 2},
$$

and thus for the second derivative in this limit we obtain

$$
S_{f}^{\prime \prime} \approx \frac{(9 \omega+1)(3 \omega+1)(\omega+1)}{2} a^{9 \omega-1 / 2}
$$

This implies $\omega<1$ for the dominating background component. This is due to the fact that

$$
\frac{S_{f}^{\prime}}{S_{h}^{\prime}} \sim \frac{S_{f}^{\prime \prime}}{S_{h}^{\prime \prime}} \longrightarrow a^{3(\omega-1) / 2} \Longrightarrow \omega<1 .
$$

Now, it is clear that in the limit of $-1 \leq \omega \leq-2 / 3$, the background piece of entropy dominates and is responsible for satisfying the GSL of thermodynamics. From (18) and (20), in the high density limit, one finds condition $\omega \leq-3 / 2$ for satisfying the GSL, which is compatible with phantom regime $(\omega<-1)$.

\section{IR Deformed Horava-Liftshitz Cosmology}

Inspired by Lifshitz theory in solid state physics, Horava proposed a field theory model for a UV complete theory of gravity [60-62]. This theory is a nonrelativistic renormalizable theory of gravity and reduces to Einstein's general relativity at large scales. The theory is usually referred to as the Horava-Lifshitz (HL) theory. It has also manifested three-dimensional spatial general covariance and time reparametrization invariance. Various aspects of HL gravity have been investigated in the literature [63-69]. In HL theory, that reduces to Einstein's general relativity at large scales, quantum field theory definitions of time and space come over Einstein's proposal and so, in the large scales, Lorentzian symmetry is achievable. The action of HL gravity is given by [60-62]

$$
\begin{gathered}
I_{\mathrm{SH}}=\int d t d^{3} x\left(\mathscr{L}_{0}+\widetilde{\mathscr{L}}_{1}+\mathscr{L}_{m}\right), \\
\mathscr{L}_{0}=\sqrt{g} N\left\{\frac{2}{\kappa^{2}}\left(K_{i j} K^{i j}-\lambda K^{2}\right)+\frac{\kappa^{2} \mu^{2}\left(\Lambda_{W} R-3 \Lambda_{W}^{2}\right)}{8(1-3 \lambda)}\right\}, \\
\widetilde{\mathscr{L}}_{1}=\sqrt{g} N\left\{\frac{\kappa^{2} \mu^{2}(1-4 \lambda)}{32(1-3 \lambda)} R^{2}-\frac{\kappa^{2}}{2 \xi^{4}}\left(C_{i j}-\frac{\mu \xi^{2}}{2} R_{i j}\right)\right. \\
\left.\times\left(C^{i j}-\frac{\mu \xi^{2}}{2} R^{i j}\right)\right\},
\end{gathered}
$$

where $\kappa^{2}, \lambda$, and $\xi$ are dimensionless constant parameters while $\mu$ and $\Lambda_{W}$ are constant parameters with mass dimensions. Here $K_{i j}$ is the extrinsic curvature which takes the form

$$
K_{i j}=\frac{1}{2 N}\left(\dot{g}_{i j}-\nabla_{i} N_{j}-\nabla_{j} N_{i}\right),
$$

and a dot denotes a derivative with respect to $t$ and covariant derivatives defined with respect to the spatial metric $g_{i j}$. In the above action $\mathscr{L}_{m}$ stands for the lagrangian of the matter field, $R$ and $R_{i j}$ are three-dimensional spatial Ricci scalar and Ricci tensor, and $C_{i j}$ is the Cotton tensor defined as

$$
C^{i j}=\epsilon^{i k l} \nabla_{k}\left(R_{l}^{j}-\frac{1}{4} R \delta_{l}^{j}\right)=\epsilon^{i k l} \nabla_{k} R_{l}^{j}-\frac{1}{4} \epsilon^{i k j} \partial_{k} R,
$$

where $\epsilon^{i k l}$ is the totally antisymmetric unit tensor. It is worth mentioning that the IR vacuum of this theory is an anti de Sitter (AdS) spacetime. Hence, it is interesting to take a limit of the theory, which may lead to a Minkowskian spacetime in the IR sector. For this purpose, one may modify the theory by introducing $\mu^{4} R$ and then take the $\Lambda_{W} \rightarrow 0$ limit [70]. This does not alter the UV properties of the theory, but it changes the IR properties. That is, there exists a Minkowski vacuum, instead of an AdS vacuum. We will now consider the limit of this theory such that $\Lambda_{W} \rightarrow 0$. The deformed action of the nonrelativistic renormalizable gravitational theory is given by [70]:

$$
\begin{aligned}
I_{\mathrm{SH}}=\int d t d^{3} x \sqrt{g} N\left\{\frac{2}{\kappa^{2}}\left(K_{i j} K^{i j}-\lambda K^{2}\right)-\frac{\kappa^{2}}{2 \xi^{4}} C_{i j} C^{i j}\right. \\
-\frac{\mu^{2} \kappa^{2}}{8} R_{i j} R^{i j}+\frac{\kappa^{2} \mu}{2 \xi^{2}} \epsilon^{i j k} R_{i l} \nabla_{j} R_{k}^{l} \\
\left.+\frac{\kappa^{2} \mu^{2}(1-4 \lambda)}{32(1-3 \lambda)} R^{2}+\mu^{4} R\right\} .
\end{aligned}
$$

In the IR limit, action (29) can be written as the standard Einstein-Hilbert action in the ADM formalism provided [70]

$$
\lambda=1, \quad c^{2}=\frac{\kappa^{2} \mu^{4}}{2}, \quad G=\frac{\kappa^{2}}{32 \pi c} .
$$

The constant $\xi$ is given by [71]

$$
\xi \equiv \frac{8 \mu^{2}(3 \lambda-1)}{\kappa^{2}} .
$$

Besides, for $\xi \rightarrow \infty$ (equivalently, $\kappa^{2} \rightarrow 0$ ), action (29) reduces to the action of Einstein gravity.

The Friedmann equation, resulting from variation of action (29) with respect to FRW metric, turns out to be [70]

$$
H^{2}=\frac{\kappa^{2}}{6(3 \lambda-1)}\left(\rho-\frac{6 k \mu^{4}}{a^{2}}-\frac{3 k^{2} \kappa^{2} \mu^{2}}{8(3 \lambda-1) a^{4}}\right) .
$$

For $k=0$, there is no contribution from the higher order derivative terms in the action. However, for $k \neq 0$, the higher derivative terms are significant for small volume, that is, for small $a$, and become insignificant for large $a$, where it agrees with general relativity. The standard Friedmann equation is recovered, in units where $c=1$, provided we define $[68,69]$

$$
\begin{gathered}
G_{\operatorname{cosm}}=\frac{\kappa^{2}}{16 \pi(3 \lambda-1)}, \\
\frac{\kappa^{2} \mu^{4}}{3 \lambda-1}=1,
\end{gathered}
$$


where condition (34) also agrees for $\lambda=1=c$ with second relation in (30). Here $G_{\text {cosm }}$ is the "cosmological" Newton's constant. In the IR limit where $\lambda=1$ the Lorentz invariance is restored, and hence $G_{\text {cosm }}=G$. Using the above identifications, as well as definition (31), the Friedmann equation (32) can be rewritten as

$$
H^{2}+\frac{k}{a^{2}}=\frac{8 \pi G_{\operatorname{cosm}}}{3} \rho+\frac{k^{2}}{2 \xi a^{4}} .
$$

One can easily see that in the limit $\xi \rightarrow \infty$ the dark radiation term vanishes and the standard Friedmann equation is restored for $\lambda=1\left(G_{\text {cosm }}=G\right)$, as expected. For a flat universe $(k=0)$, this equation reduces to

$$
H^{2}=\frac{8 \pi G_{\operatorname{cosm}}}{3} \rho .
$$

In the deformed HL gravity, the entropy associated with the event horizon of a static spherically symmetric black hole takes the form [72]:

$$
S_{h}=\frac{A}{4 G}+\frac{\pi}{\xi} \ln \frac{A}{G},
$$

where $A=4 \pi r_{+}^{2}$ is the area of the black hole horizon and $k_{B}=$ $c=\hbar=1$ set for simplicity. Replacing $r_{+}$with the Hubble radius $H^{-1}$ in the flat FRW Universe, we have for the entropy of IR modified HL cosmology:

$$
S_{h}=\frac{\pi H^{-2}}{G}+\frac{\pi}{\xi} \ln \frac{4 \pi H^{-2}}{G} .
$$

Taking the first derivative of the entropy with respect to scale factor, one gets

$$
S_{h}^{\prime}=\frac{3(\omega+1)}{a}\left[\frac{\pi}{\xi}+\frac{3}{8 G^{2}} a^{3(\omega+1)}\right],
$$

where we have used

$$
\frac{d \ln H}{d \ln a}=-\frac{3(\omega+1)}{2}
$$

and relation (36). For the second derivative of entropy, we have

$$
S_{h}^{\prime \prime}=\frac{3(\omega+1)}{a^{2}}\left[-\frac{\pi}{\xi}+\frac{3}{8 G^{2}}(3 \omega+2) a^{3(\omega+1)}\right] .
$$

In the long run limit where $a \rightarrow \infty$, we have $3 \omega+3 \geq 0$, and so we arrive at

$$
\begin{gathered}
S_{h}^{\prime} \approx(\omega+1) a^{3 \omega+2}, \\
S_{h}^{\prime \prime} \approx(\omega+1)(3 \omega+2) a^{3 \omega+1} .
\end{gathered}
$$

These results imply the range $(-1 \leq \omega \leq-2 / 3)$ for satisfying $S_{h}^{\prime} \geq 0$ and $S_{h}^{\prime \prime} \leq 0$. For the fluid which is enveloped by the Hubble Horizon, we get

$$
\begin{gathered}
S_{f}^{\prime}=\frac{9(\omega+1)(3 \omega+1) a^{9 \omega+5} \rho_{0}}{32 \pi G^{2} T_{0}}, \\
S_{f}^{\prime \prime}=\frac{9(\omega+1)(3 \omega+1)(9 \omega+5) a^{9 \omega+4} \rho_{0}}{32 \pi G^{2} T_{0}} .
\end{gathered}
$$

The ratio between the fluid and the horizon entropy is given by

$$
\frac{S_{f}^{\prime \prime}}{S_{h}^{\prime \prime}} \simeq \frac{S_{f}^{\prime}}{S_{h}^{\prime}} \sim a^{6 \omega+3},
$$

which implies $\omega<-1 / 2$, for dominating spacetime entropy. By comparing this condition with what we saw from (42), we get

$$
-1 \leq \omega \leq-\frac{2}{3},
$$

as a proper domain. Another condition arises from our second assumption which implies $3 \omega+3 \geq 0$. If we consider opposite statement $(3 \omega+3<0)$ then for satisfying the GSL by background fluid we find $\omega<-1$, which is consistent with (44).

\section{DGP Braneworld Model}

For spatially flat FRW background, the Friedmann equation in DGP braneworld can be written as $[73,74]$

$$
H^{2}=\left(\sqrt{\frac{\mu^{2} \rho}{3}+\frac{1}{4 r_{c}^{2}}}+\frac{\epsilon}{2 r_{c}}\right)^{2},
$$

where $\mu^{2}=8 \pi G_{4}, r_{c}=M_{p}^{2} / 2 M_{5}^{2}$ is the crossover scale which determines the transition from $4 D$ to $5 D$ behavior, and $\epsilon= \pm 1$ corresponds to the two branches of the DGP braneworld [75]. Equation (46) with $\epsilon=1$ and $\rho=0$ has an interesting selfaccelerating solution with a Hubble parameter given by the inverse of the crossover scale $r_{c}$ [76]. It was shown in [77] that there are some cosmological constraints that confine this model beside its prediction about cross over scale which is inconsistent with reality (because its scale is of the order of the Solar system). However, it has attracted some investigations. The reason of these attempts comes from its view about acceleration which have been argued in ample details in the literatures [78-80]. Using (2) and (46) it is clear that in the low densities the condition $\ddot{a} \geq 0$ is satisfied, independent of $\omega$. For high densities, $\omega \leq-1 / 3$ emerges as an upper bound for $\omega$. The entropy associated with the apparent horizon in this model is given by [81]

$$
S_{h}=\frac{A}{4 G_{4}}\left(1-\frac{\epsilon \widetilde{r}_{A}}{3 r_{c}}\right) \text {. }
$$

It is obvious that, for $\epsilon=-1$ entropy is positive, whereas for $\epsilon=+1$, the positivity of entropy implies that $\widetilde{r}_{A}<3 r_{c}$, which is consistent with numerical simulations [77]. By evaluating the first derivative of the entropy, we find

$$
\begin{aligned}
S_{h}^{\prime}= & \frac{2 \pi \mu^{2}(\omega+1) \rho}{a G_{4} \sqrt{\mu^{2} \rho / 3+1 /\left(2 r_{c}\right)^{2}}\left(\sqrt{\mu^{2} \rho / 3+1 /\left(2 r_{c}\right)^{2}}+\epsilon\left(1 / 2 r_{c}\right)\right)^{3}} \\
& \times\left(1-\left(\epsilon \tilde{r}_{A} / 2 r_{c}\right)\right) .
\end{aligned}
$$


For $\epsilon=-1$ the above expression will be nonnegative provided that $\omega \geq-1$. For positive value of $\epsilon$, the condition $S_{h}^{\prime} \geq 0$ yields a similar result for $\widetilde{r}_{A}<2 r_{c}$, and phantom regime for $2 r_{c}<\widetilde{r}_{A}<3 r_{c}$. In the limit of $a \rightarrow \infty$, one gets

$$
S_{h}^{\prime} \approx \frac{3(\omega+1)}{\mu^{2}} a^{3 \omega+2}\left(1-\frac{\epsilon \sqrt{3}}{2 r_{c} \mu} a^{-3((\omega+1) / 2)}\right) .
$$

For nonphantom regime and independent of the value of $\epsilon$, we get $S_{h}^{\prime} \approx(\omega+1) a^{(3 \omega+2)}$ and $S_{h}^{\prime \prime} \approx(\omega+1)(3 \omega+2) a^{3 \omega+1}$, which yields $-1 \leq \omega \leq-2 / 3$ as a proper domain for satisfying GSL by background. For the entropy of fluid, from (11) we find

$$
S_{f}^{\prime}=\frac{3 V \rho(\omega+1)}{a T}\left(\frac{\mu^{2} \rho}{H \sqrt{\mu^{2} \rho / 3+1 / 4 r_{c}^{2}}}-1\right) \text {, }
$$

where in the long run limit yields

$$
\begin{aligned}
& S_{f}^{\prime} \approx a^{(9 \omega+1) / 2}, \\
& S_{f}^{\prime \prime} \approx a^{(9 \omega-1) / 2} .
\end{aligned}
$$

The ratio between fluid and horizon entropy is given by

$$
\frac{S_{f}^{\prime \prime}}{S_{h}^{\prime \prime}} \simeq \frac{S_{f}^{\prime}}{S_{h}^{\prime}} \approx a^{(3 \omega-3) / 2} \Longrightarrow \omega<1 .
$$

Comparing this condition with one previously obtained for the satisfaction of the GSL, it becomes clear that, for $-1 \leq$ $\omega \leq-2 / 3$, the DGP braneworld model approaches thermal equilibrium in the late time, which is consistent with observations at the present time $[41,42]$. In the phantom regime where $2 r_{c}<\tilde{r}_{A}<3 r_{c}, \omega<-1$, the ratio between fluid and horizon entropy is given by

$$
\frac{S_{f}^{\prime \prime}}{S_{h}^{\prime \prime}} \simeq \frac{S_{f}^{\prime}}{S_{h}^{\prime}} \approx a^{3 \omega} \Longrightarrow \omega<0 .
$$

By calculating the asymptotic behavior of the entropy derivatives in this regime, namely, $S_{h}^{\prime} \simeq-(\omega+1) a^{(3 \omega+1) / 2}, S_{h}^{\prime \prime} \simeq$ $-(\omega+1)(3 \omega+1) a^{(3 \omega-1) / 2}$, and considering the results of (53) we get $\omega<-1$.

\section{Gauss Bonnet Gravity}

The action of Gauss-Bonnet gravity in $(n+1)$ dimensions can be written as

$$
I=\frac{1}{16 \pi G} \int d^{n+1} x \sqrt{-g}\left(R+\alpha \mathscr{L}_{\mathrm{GB}}\right)+I_{m},
$$

where $\mathscr{L}_{\mathrm{GB}}=R^{2}-4 R_{\mu \nu} R^{\mu \nu}+R_{\mu \nu \lambda \rho} R^{\mu \nu \lambda \rho}$ is the lagrangian of the Gauss-Bonnet correction term, $\alpha$ is the Gauss-Bonnet coefficient which is a positive constant, and $I_{m}$ denotes the matter action. The Gauss-Bonnet term is a topological term in four dimensions, and its expansion around flat spacetime is ghost-free. Although the action includes higher order derivatives of curvature terms, there are no more than second-order derivatives of metric in the equation of motion. The entropy of the static spherically symmetric black hole in $(n+1)$-dimensional Gauss-Bonnet theory has the following form [82-86]:

$$
S_{h}=\frac{A}{4 G}\left(1+\frac{n-1}{n-3} \frac{2 \widetilde{\alpha}}{r_{+}^{2}}\right) \text {, }
$$

where $\widetilde{\alpha}=(n-2)(n-3) \alpha$ and $A=n \Omega_{n} r_{+}^{n-1}$. Taking into account the entropy formula (55) for the apparent horizon, using the apparent horizon radius $\widetilde{r}_{A}$ instead of the black hole radius $r_{+}$, and applying the first law, one obtains the Friedmann equation in the Gauss-Bonnet cosmology as [9]

$$
H^{2}+\tilde{\alpha} H^{4}=\frac{16 \pi G}{n(n-1)} \rho .
$$

Taking the derivative with respect to scale factor $a$ and using relation (6), one finds

$$
\frac{d H}{d a}=-\frac{n(\omega+1) \beta \rho}{2 a\left[H+2 \widetilde{\alpha} H^{3}\right]},
$$

where $\beta=16 \pi G / n(n-1)$. After inserting (57) into (2) and doing simple calculations, one arrives at

$$
\omega \leq \frac{2 \widetilde{\alpha} H^{4}}{n \beta \rho}+\frac{2-n}{n},
$$

where $H$ obviously decreases by expansion [87-89]; therefore, for small values of $H$ (late time limit), this reduces to

$$
\omega \leq \frac{2}{n}\left(\tilde{\alpha} \beta \rho+1-\frac{n}{2}\right) .
$$

As a proper upper bound for density, one can consider $(2 \tilde{\alpha} \beta \rho / n+(2-n) / n)$ in which we have used $H^{2} \sim \beta \rho$. Taking the first derivative of the entropy of the apparent horizon with respect to the scale factor, we find

$$
S_{h}^{\prime}=\frac{n^{2}(n-1) \Omega_{n}(\omega+1) \beta \rho \widetilde{r}_{A}^{n}}{8 G a H},
$$

where we have used

$$
\widetilde{r}_{h}^{\prime}=\frac{d \widetilde{r}_{A}}{d a}=\frac{d \widetilde{r}_{A}}{d H} \frac{d H}{d a}=\frac{n(\omega+1) \beta \rho}{2 a H\left[H^{2}+2 \widetilde{\alpha} H^{4}\right]} .
$$

Employing the second law of thermodynamics, we get $w \geq$ -1 , which implies a decreasing density, $\rho=\rho_{0} a^{-n(\omega+1)}$, in the long run limit for negative values of $\omega$. Therefore, we can neglect the effects of higher orders of $H$ in the left hand side of (56) and justify relation $H^{2} \simeq \beta \rho$. By evaluating the second derivative we get

$$
\begin{aligned}
S_{h}^{\prime \prime}= & \frac{n^{2}(n-1) \Omega_{n}(\omega+1) \beta \rho}{8 G a^{2} H^{n+1}} \\
& \times\left[-(1+n \omega+n)+a H \widetilde{r}_{A}^{\prime}(n+1)\right] .
\end{aligned}
$$


In the long run limit $\widetilde{r}_{A}^{\prime} \simeq n(\omega+1) / 2 a H$ and we have

$$
S_{h}^{\prime \prime} \approx(\omega+1)\left[(\omega+1)\left(n^{2}-n\right)-2\right] \frac{\rho}{a^{2} H^{n+1}} .
$$

Using the GSL of thermodynamics we find $-1 \leq \omega \leq-1+$ $2 /\left(n^{2}-n\right)$, which shows that the GSL can be satisfied by the background fluid. Note that $\omega=-1$ and $\omega=-1+2 /\left(n^{2}-n\right)$ are roots of equation $S_{h}^{\prime \prime}=0$, and therefore condition $S_{h}^{\prime \prime} \leq 0$ is satisfied. Also, by using the result of applying the second law to (60) which includes $\omega \geq-1$, it is obvious that GSL is satisfied. For $n=3$, the result is the same as Einstein's gravity [39]. By increasing $n, \omega$ approaches -1 as a limiting value. Using the Gibbs' law for the fluid, we obtain

$$
S_{f}^{\prime}=\frac{n(\omega+1) V_{n} \rho_{0}}{a^{n+1} T_{0}}\left[\frac{n(\omega+1) \beta \rho}{2\left(H^{2}+2 \widetilde{\alpha} H^{4}\right)}-1\right] .
$$

Thus, in the long run limit we have $S_{f}^{\prime} \sim((n(\omega+1)-2) /$ 2) $a^{\left(n^{2}(\omega+1)-2 n-2\right) / 2}$ and $S_{f}^{\prime \prime} \sim a^{\left(n^{2}(\omega+1)-2 n-4\right) / 2}$. The relations between various components of entropy are as follows:

$$
\frac{S_{f}^{\prime \prime}}{S_{h}^{\prime \prime}} \simeq \frac{S_{f}^{\prime}}{S_{h}^{\prime}} \sim a^{-(n / 2)(\omega+3)} \Longrightarrow w>-3 .
$$

This result indicates $-1 \leq \omega \leq-1+2 /\left(n^{2}-n\right)$ as a proper domain for satisfying the GSL in Gauss-Bonnet gravity.

\section{Conclusions and Discussion}

The GSL of thermodynamics in cosmology not only implies that the entropy of our Universe must be always a nondecreasing function of scale factor $S^{\prime} \geq 0$, but also it must be a convex function, $S^{\prime \prime} \leq 0$, where the prime represents derivative with respect to the scale factor $a$. Inspired by these requirements, we investigated the total entropy within the Hubble horizon in various models of cosmology. The satisfaction of these conditions put upper and lower bounds on the equation of state parameter $\omega$ of each cosmological model. We considered a cosmological background filled with a fluid with the linear equation of state $p=\omega \rho$. We exploited the entropy conditions and studied the long run limit to derive the proper domain of the $\omega$ parameter in various models of cosmology including ghost dark energy, cyclic universe model, IR modified HL cosmology, DGP braneworld, and Gauss-Bonnet cosmology. The present investigation extends the results obtained earlier and confirms that the acceptable range of the equation of state parameter is severely restricted if the GSL remains to be valid. Our investigations show that the satisfaction of the GSL of thermodynamics in various cosmological models requires the existence of some kind of energy in our Universe with $\omega<-1 / 3$. In other words, the existence of a dark energy component or equivalently modified gravity theory, is unavoidable if we require the cosmological models to approach thermal equilibrium in the long run limit.

\section{Conflict of Interests}

The authors declare no conflict of interests for the present paper.

\section{Acknowledgments}

A. Sheykhi thanks the Research Council of Shiraz University. The work of A. Sheykhi has been supported financially by Research Institute for Astronomy and Astrophysics of Maragha (RIAAM), Iran.

\section{References}

[1] T. Jacobson, "Thermodynamics of spacetime: the Einstein equation of state," Physical Review Letters, vol. 75, no. 7, pp. 12601263, 1995.

[2] C. Eling, R. Guedens, and T. Jacobson, "Nonequilibrium thermodynamics of spacetime," Physical Review Letters, vol. 96, no. 12, Article ID 121301, 4 pages, 2006.

[3] T. Padmanabhan, "Classical and quantum thermodynamics of horizons in spherically symmetric spacetimes," Classical and Quantum Gravity, vol. 19, no. 21, pp. 5387-5408, 2002.

[4] T. Padmanabhan, "Gravity and the thermodynamics of horizons," Physics Reports, vol. 406, no. 2, pp. 49-125, 2005.

[5] T. Padmanabhan, "Gravity: a new holographic perspective," International Journal of Modern Physics D, vol. 15, no. 10, p. 1659, 2006.

[6] M. Akbar and R. G. Cai, "Friedmann equations of FRW universe in scalar-tensor gravity, $f(R)$ gravity and first law of thermodynamics," Physics Letters B, vol. 635, no. 1, pp. 7-10, 2006.

[7] R. G. Cai and L. M. Cao, "Unified first law and the thermodynamics of the apparent horizon in the FRW universe," Physical Review D, vol. 75, no. 6, Article ID 064008, 11 pages, 2007.

[8] M. Akbar and R. G. Cai, "Thermodynamic behavior of the Friedmann equation at the apparent horizon of the FRW universe," Physical Review D, vol. 75, no. 8, Article ID 084003, 9 pages, 2007.

[9] R. G. Cai and S. P. Kim, "First law of thermodynamics and Friedmann equations of Friedmann-Robertson-Walker universe," Journal of High Energy Physics, vol. 2005, no. 2, article 50, 2005.

[10] E. Verlinde, "On the origin of gravity and the laws of Newton," Journal of High Energy Physics, vol. 2011, no. 4, article 29, 2011.

[11] R. G. Cai, L. M. Cao, and N. Ohta, "Friedmann equations from entropic force," Physical Review D, vol. 81, no. 6, Article ID 061501, 2010.

[12] R. G. Cai, L. M. Cao, and N. Ohta, "Notes on entropy force in general spherically symmetric spacetimes," Physical Review D, vol. 81, no. 8, Article ID 084012, 9 pages, 2010.

[13] Y. S. Myung and Y. W. Kim, "Entropic force and entanglement system," Physical Review D, vol. 81, no. 10, Article ID 105012, 7 pages, 2010.

[14] R. Banerjee and B. R. Majhi, "Statistical origin of gravity," Physical Review D, vol. 81, no. 12, Article ID 124006, 5 pages, 2010.

[15] S. W. Wei, Y. X. Liu, and Y. Q. Wang, "A note on Friedmann equation of FRW universe in deformed Hořava-Lifshitz gravity from entropic force," Communications in Theoretical Physics, vol. 56, no. 3, article 455, 2011.

[16] Y. X. Liu, Y. Q. Wang, and S. W. Wei, "A note on the temperature and energy of four-dimensional black holes from an entropic force," Classical and Quantum Gravity, vol. 27, no. 18, Article ID 185002, 2010.

[17] R. A. Konoplya, "Entropic force, holography and thermodynamics for static space-times," European Physical Journal C, vol. 69, no. 3-4, pp. 555-562, 2010. 
[18] H. Wei, "Cosmological constraints on the modified entropic force model," Physics Letters B, vol. 692, no. 3, pp. 167-175, 2010.

[19] C. M. Ho, D. Minic, and Y. J. Ng, "Cold dark matter with MOND scaling," Physics Letters B, vol. 693, no. 5, pp. 567-570, 2010.

[20] V. V. Kiselev and S. A. Timofeev, "The holographic screen at low temperatures," Modern Physics Letters A, vol. 26, no. 2, p. 109, 2011.

[21] W. Gu, M. Li, and R. X. Miao, "A new entropic force scenario and holographic thermodynamics," Science China Physics, Mechanics and Astronomy, vol. 54, no. 11, pp. 1915-1924, 2011.

[22] R. X. Miao, J. Meng, and M. Li, “ $f(R)$ gravity and Maxwell equations from the holographic principle," Science China Physics, Mechanics and Astronomy, vol. 55, no. 3, pp. 375-380, 2012.

[23] A. Sheykhi, "Entropic corrections to Friedmann equations," Physical Review D, vol. 81, no. 10, Article ID 104011, 4 pages, 2010.

[24] Y. Ling and J. P. Wu, "A note on entropic force and brane cosmology," Journal of Cosmology and Astroparticle Physics, vol. 2010, no. 8, article 17, 2010.

[25] L. Modesto and A. Randono, "Entropic corrections to Newton's law," http://arxiv.org/abs/1003.1998.

[26] L. Smolin, "Newtonian gravity in loop quantum gravity," http:// arxiv.org/abs/1001.3668.

[27] X. Li and Z. Chang, "Debye Entropic Force and Modified Newtonian Dynamics," Communications in Theoretical Physics, vol. 55, no. 4, article 733, 2011.

[28] Y. F. Cai, J. Liu, and H. Li, "Entropic cosmology: a unified model of inflation and late-time acceleration," Physics Letters B, vol. 690, no. 3, pp. 213-219, 2010.

[29] M. Li and Y. Wang, "Quantum UV/IR relations and holographic dark energy from entropic force," Physics Letters B, vol. 687, no. 2-3, pp. 243-247, 2010.

[30] S. H. Hendi and A. Sheykhi, "Entropic corrections to Einstein equations," Physical Review D, vol. 83, no. 8, Article ID 084012, 5 pages, 2011.

[31] A. Sheykhi and S. H. Hendi, "Power-law entropic corrections to Newton's law and Friedmann equations," Physical Review D, vol. 84, no. 4, Article ID 044023, 8 pages, 2011.

[32] S. H. Hendi and A. Sheykhi, "Entropic Corrections to Coulomb's Law," International Journal of Theoretical Physics, vol. 51, no. 4, pp. 1125-1136, 2012.

[33] A. Sheykhi and Z. Teimoori, "Modified Friedmann equations from Debye entropic gravity," General Relativity and Gravitation, vol. 44, no. 5, pp. 1129-1141, 2012.

[34] A. Sheykhi, "Modified Friedmann equations on the brane from entropic force," International Journal of Theoretical Physics, vol. 51, no. 1, pp. 185-192, 2012.

[35] A. Sheykhi, H. Moradpour, and N. Riazi, "Lovelock gravity from entropic force," General Relativity and Gravitation, vol. 45, no. 5, pp. 1033-1049, 2013.

[36] H. B. Callen, Thermodynamics and Introduction to Thermostatics, John Wiley \& Sons, New York, NY, USA, 1985.

[37] H. B. Callen, Thermodynamics, John Wiley \& Sons, New York, NY, USA, 1960.

[38] N. Radicella and D. Pavón, "Thermodynamical analysis on a braneworld scenario with curvature corrections," Physics Letters $B$, vol. 704, no. 4, pp. 260-264, 2011.

[39] N. Radicella and D. Pavón, "A thermodynamic motivation for dark energy," General Relativity and Gravitation, vol. 44, no. 3, pp. 685-702, 2012.
[40] B. Wang, E. Abdalla, and T. Osada, "Behavior of shell effects with the excitation energy in atomic nuclei," Physical Review Letters, vol. 85, no. 1, pp. 26-29, 2000.

[41] S. Perlmutter, G. Aldering, G. Goldhaber et al., "Measurements of $\Omega$ and $\Lambda$ from 42 high-redshift supernovae," The Astrophysical Journal, vol. 517, no. 2, article 565, 1999.

[42] A. G. Riess, A. V. Filippenko, P. Challis et al., "Observational evidence from supernovae for an accelerating universe and a cosmological constant," The Astronomical Journal, vol. 116, no. 3, article 1009, 1998.

[43] M. Jones, H. Robert, and J. Lambourne, An Introduction to Galaxies and Cosmology, Cambridge University Press, New York, NY, USA, 2004.

[44] N. Ohta, "Dark energy and QCD ghost," Physics Letters B, vol. 695, no. 1-4, pp. 41-44, 2011.

[45] R. G. Cai, Z. L. Tuo, and H. B. Zhang, "Notes on ghost dark energy," Physical Review D, vol. 84, no. 12, Article ID 123501, 10 pages, 2011.

[46] R. G. Cai, Z. L. Tuo, Y. B. Wu, and Y. Y. Zhao, "More on QCD ghost dark energy," Physical Review D, vol. 86, no. 2, Article ID 023511, 8 pages, 2012.

[47] A. Sheykhi and A. Bagheri, "Quintessence ghost dark energy model," Europhysics Letters, vol. 95, no. 3, Article ID 39001, 2011.

[48] A. Sheykhi and M. S. Movahed, "Interacting ghost dark energy in non-flat universe," General Relativity and Gravitation, vol. 44, no. 2, pp. 449-465, 2012.

[49] E. Ebrahimi and A. Sheykhi, "Interacting ghost dark energy in Brans-Dicke theory," Physics Letters B, vol. 705, no. 1, pp. 19-25, 2011.

[50] E. Ebrahimi and A. Sheykhi, "Instability of QCD ghost dark energy model," International Journal of Modern Physics D, vol. 20, no. 12, p. 2369, 2011.

[51] A. Sheykhi, M. S. Movahed, and E. Ebrahimi, "Tachyon reconstruction of ghost dark energy," Astrophysics and Space Science, vol. 339, no. 1, pp. 93-99, 2012.

[52] F. R. Urban and A. R. Zhitnitsky, “The cosmological constant from the QCD Veneziano ghost," Physics Letters B, vol. 688, no. 1, pp. 9-12, 2010.

[53] K. Kawarabayashi and N. Ohta, "The $\eta$ problem in the large- $N$ limit: effective Lagrangian approach," Nuclear Physics B, vol. 175, no. 3, pp. 477-492, 1980.

[54] J. D. Bekenstein, "Black Holes and Entropy," Physical Review D, vol. 7, no. 8, pp. 2333-2346, 1973.

[55] G. Izquierdo and D. Pavón, "Dark energy and the generalized second law," Physics Letters B, vol. 633, no. 4-5, pp. 420-426, 2006.

[56] M. O. Calvão, J. A. S. Lima, and I. Waga, "On the thermodynamics of matter creation in cosmology," Physics Letters A, vol. 162, no. 3, pp. 223-226, 1992.

[57] Y. Shtanov and V. Sahni, "Bouncing braneworlds," Physics Letters $B$, vol. 557, no. 1-2, pp. 1-6, 2003.

[58] A. Ashtekar, T. Pawlowski, and P. Singh, "Quantum nature of the big bang: an analytical and numerical investigation," Physical Review D, vol. 73, no. 12, Article ID 124038, 33 pages, 2006.

[59] X. Zhang, "Can black holes be torn up by phantom dark energy in cyclic cosmology?" The European Physical Journal C, vol. 60, no. 4, pp. 661-667, 2009.

[60] P. Horava, "Quantum gravity at a Lifshitz point," Physical Review $D$, vol. 79, no. 8, Article ID 084008, 15 pages, 2009.

[61] P. Horava, "Membranes at quantum criticality," Journal of High Energy Physics, vol. 2009, no. 3, article 20, 2009. 
[62] P. Horava, "Spectral dimension of the universe in quantum gravity at a Lifshitz point," Physical Review Letters, vol. 102, no. 16, Article ID 161301, 4 pages, 2009.

[63] Y. S. Myung, "Thermodynamics of black holes in the deformed Hořava-Lifshitz gravity," Physics Letters $B$, vol. 678, no. 1, pp. 127-130, 2009.

[64] R. B. Mann, "Lifshitz topological black holes," Journal of High Energy Physics, vol. 2009, no. 6, article 75, 2009.

[65] R. G. Cai, L. M. Cao, and N. Ohta, "Thermodynamics of black holes in Hořava-Lifshitz gravity," Physics Letters B, vol. 679, no. 5, pp. 504-509, 2009.

[66] Y.S. Myung, "Generalized uncertainty principle, quantum gravity and Hořava-Lifshitz gravity," Physics Letters B, vol. 681, no. 1, pp. 81-84, 2009.

[67] R. G. Cai and N. Ohta, "Horizon thermodynamics and gravitational field equations in Hořava-Lifshitz gravity," Physical Review $D$, vol. 81, no. 8, Article ID 084061, 8 pages, 2010.

[68] M. Jamil, E. N. Saridakis, and M. R. Setare, "The generalized second law of thermodynamics in Hořava-Lifshitz cosmology," Journal of Cosmology and Astroparticle Physics, vol. 2010, no. 11, article 32, 2010.

[69] A. Sheykhi, "Thermodynamics of the apparent horizon in infrared modified Hořava-Lifshitz gravity," Physical Review D, vol. 87, no. 2, Article ID 024022, 7 pages, 2013.

[70] A. Kehagias and K. Sfetsos, "The black hole and FRW geometries of non-relativistic gravity," Physics Letters B, vol. 678, no. 1, pp. 123-126, 2009.

[71] A. Castillo and A. Larranaga, "Entropy for black holes in the deformed Horava-Lifshitz gravity," Electronic Journal of Theoretical Physics, vol. 8, pp. 1-10, 2011.

[72] Y. S. Myung, "Entropy of black holes in the deformed HořavaLifshitz gravity," Physics Letters B, vol. 684, no. 2-3, pp. 158-161, 2010.

[73] G. R. Dvali, G. Gabadadze, and M. Porrati, "4D gravity on a brane in 5D Minkowski space," Physics Letters B, vol. 485, no. 1-3, pp. 208-214, 2000.

[74] A. Sheykhi, M. H. Dehghani, and S. E. Hosseini, "Emergence of spacetime dynamics in entropy corrected and braneworld models," Journal of Cosmology and Astroparticle Physics, vol. 2013, no. 4, article 38, 2013.

[75] C. Deffayet, "Cosmology on a brane in Minkowski bulk," Physics Letters B, vol. 502, no. 1-4, pp. 199-208, 2001.

[76] C. Deffayet and G. Dvali, "Accelerated universe from gravity leaking to extra dimensions," Physical Review D, vol. 65, no. 4, Article ID 044023, 9 pages, 2002.

[77] L. Lombriser, W. Hu, W. Fang, and U. Seljak, "Cosmological constraints on DGP braneworld gravity with brane tension," Physical Review D, vol. 80, no. 6, Article ID 063536, 12 pages, 2009.

[78] A. Sheykhi, B. Wang, and R. G. Cai, "Thermodynamical properties of apparent horizon in warped DGP braneworld," Nuclear Physics B, vol. 779, no. 1-2, pp. 1-12, 2007.

[79] M. H. Dehghani, S.H. Hendi, A. Sheykhi, and H. R. Sedehi, "Thermodynamics of rotating black branes in Einstein-BornInfeld-dilaton gravity," Journal of Cosmology and Astroparticle Physics, vol. 2007, no. 2, article 20, 2007.

[80] A. Sheykhi and B. Wang, "Generalized second law of thermodynamics in warped DGP braneworld," Modern Physics Letters A, vol. 25, no. 14, p. 1199, 2010.

[81] K. Nozari, N. Behrooz, and A. Sheykhi, "Thermodynamics of viscous dark energy in DGP setup," International Journal of Theoretical Physics, vol. 52, no. 7, pp. 2351-2362, 2013.
[82] R. C. Myers and J. Z. Simon, "Black-hole thermodynamics in Lovelock gravity," Physical Review D, vol. 38, no. 8, pp. 24342444, 1988.

[83] R. G. Cai, "Gauss-Bonnet black holes in AdS spaces," Physical Review D, vol. 65, no. 8, Article ID 084014, 9 pages, 2002.

[84] R. G. Cai and Q. Guo, "Gauss-Bonnet black holes in dS spaces," Physical Review D, vol. 69, no. 10, Article ID 104025, 9 pages, 2004.

[85] R. G. Cai and K. S. Soh, "Topological black holes in the dimensionally continued gravity," Physical Review D, vol. 59, no. 4, Article ID 044013, 13 pages, 1999.

[86] T. Clunan, S. F. Ross, and D. J. Smith, "On Gauss-Bonnet black hole entropy," Classical and Quantum Gravity, vol. 21, no. 14, article 3447, 2004.

[87] E. V. Linder, "Uniqueness of current cosmic acceleration," Physical Review D, vol. 82, no. 6, Article ID 063514, 6 pages, 2010.

[88] E. V. Linder and T. L. Smith, "Dark before light: testing the cosmic expansion history through the cosmic microwave background," Journal of Cosmology and Astroparticle Physics, vol. 2011, no. 4, article 1, 2011.

[89] P. Serra, A. Cooray, D. E. Holz, A. Melchiorri, S. Pandolfi, and D. Sarkar, "No evidence for dark energy dynamics from a global analysis of cosmological data," Physical Review D, vol. 80, no. 12, Article ID 121302(R), 5 pages, 2009. 

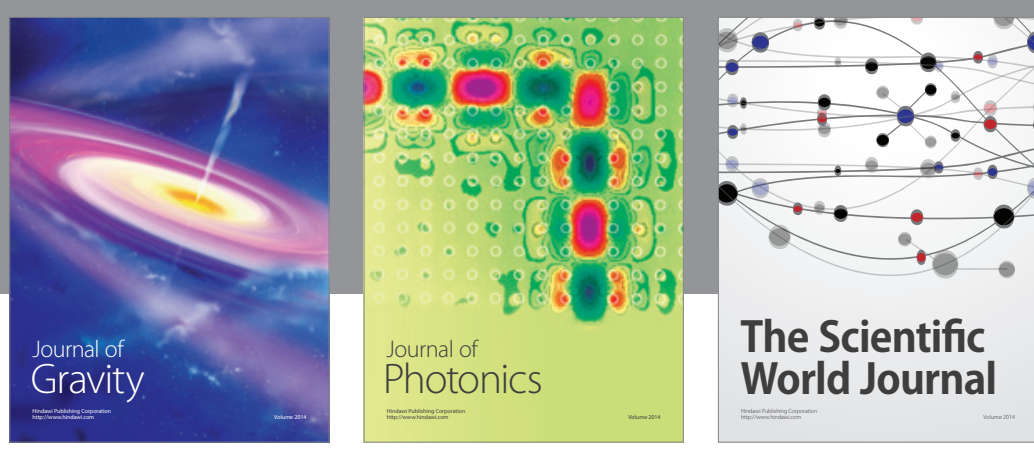

The Scientific World Journal
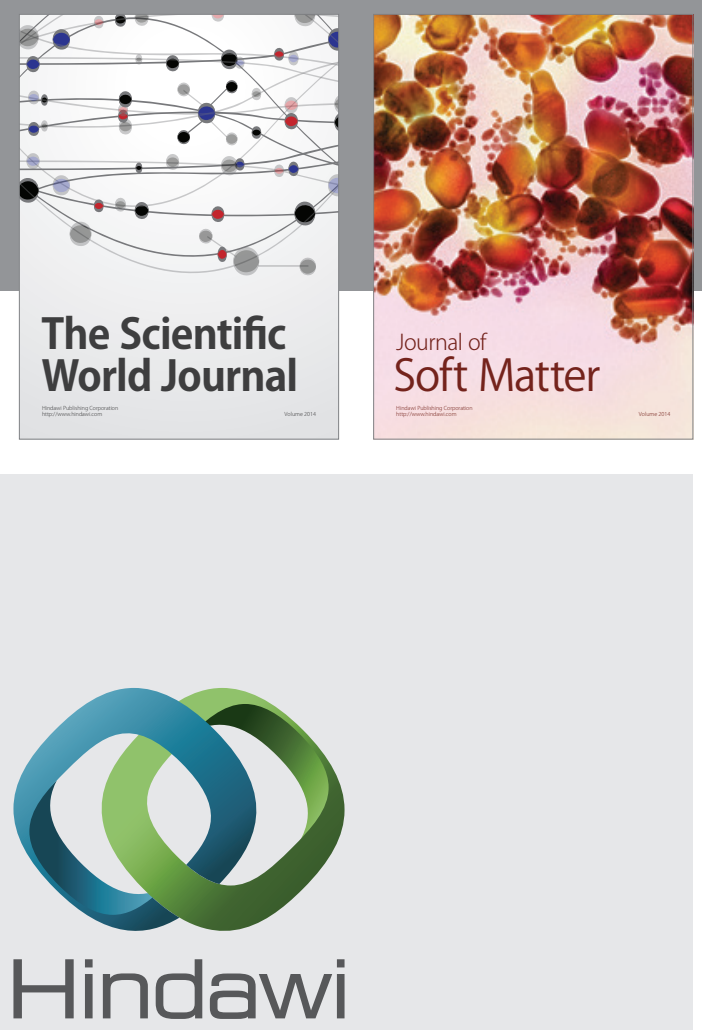

Submit your manuscripts at

http://www.hindawi.com

nternational Journal of

Statistical Mechanics
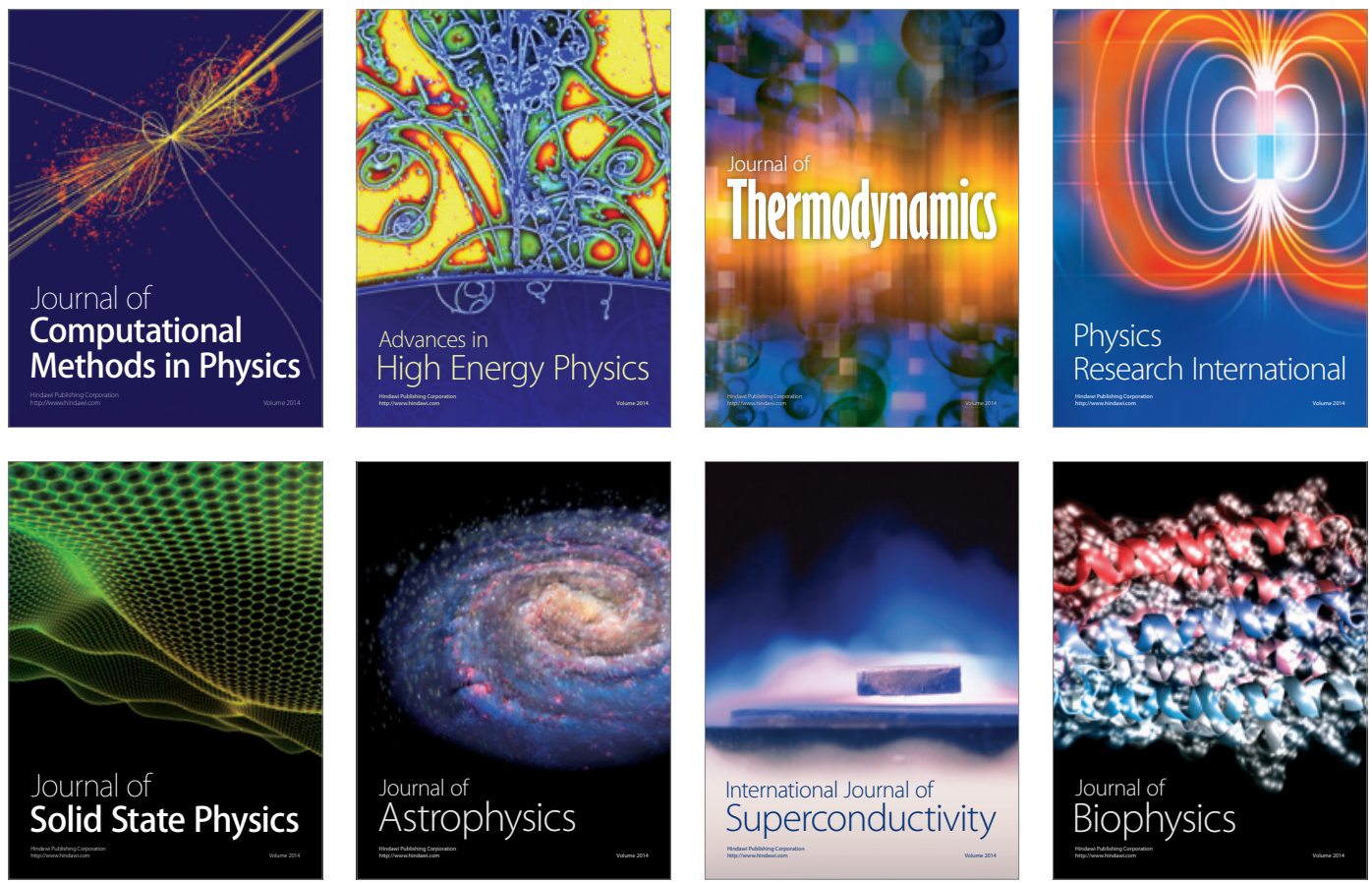
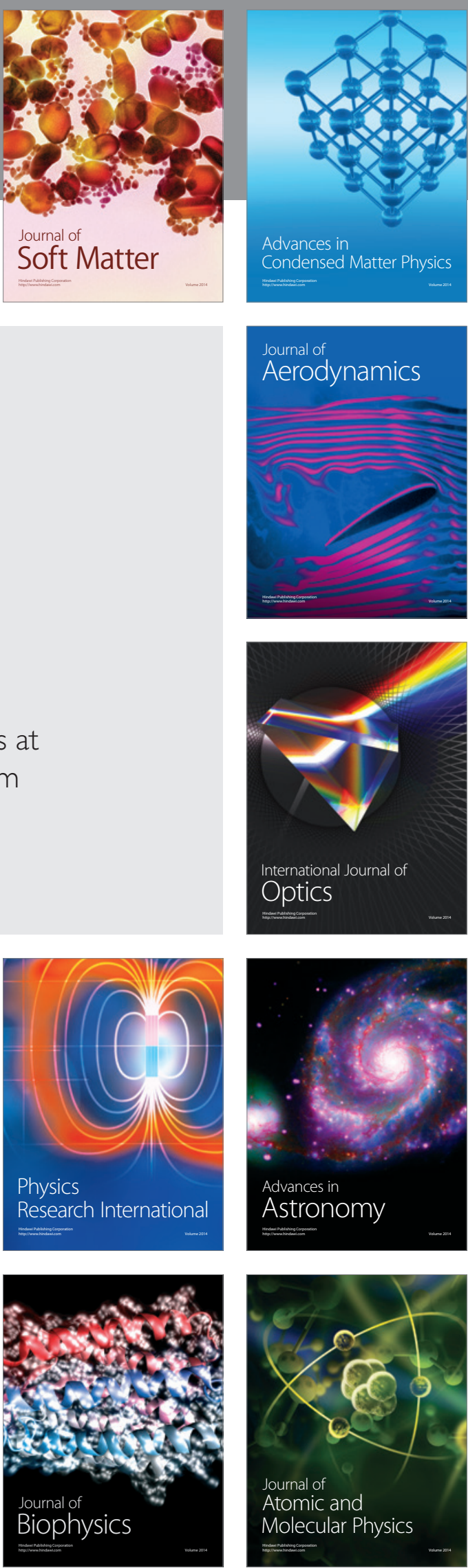